\title{
Téoros
}

Revue de recherche en tourisme

\section{Géologie, mines et tourisme en Abitibi-Témiscamingue}

\section{Guy Perreault}

Volume 15, numéro 2, été 1996

Patrimoine industriel

URI : https://id.erudit.org/iderudit/1075025ar

DOI : https://doi.org/10.7202/1075025ar

Aller au sommaire du numéro

Éditeur(s)

Université du Québec à Montréal

ISSN

0712-8657 (imprimé)

1923-2705 (numérique)

Découvrir la revue

Citer cet article

Perreault, G. (1996). Géologie, mines et tourisme en Abitibi-Témiscamingue. Téoros, 15(2), 31-35. https://doi.org/10.7202/1075025ar d'utilisation que vous pouvez consulter en ligne.

https://apropos.erudit.org/fr/usagers/politique-dutilisation/ 


\section{GÉOLOGIE, MINES ET TOURISME EN ABITIBI-TÉMISCAMINGUE}

Guy Perreault, géographe, Ph.D. (éducation)

La géologie et l'exploitation minière de l'Abitibi-Téniscaningue sont des réalités qui offrent de nombrewx points d'interêt. Pour les personnes qui visitent cette région, il est cependant difficile d'avoir un accès direct aux installations. Hewreusenent, de nombreuses personnes et plusieurs groupes s'intéressent depuis plusieurs années à meltre en valeur ces ressources et leur histoire. Le visite ar peut maintenant s'autarder à au moins trois endroits bien equipés pour le recevoir et l'intúresser. Dans la prenière partie de cet article, je rappelle quelques notions de géologie afin de wieut comprendre l'origine et la valeur des ressources minières qui sont non renouvelables. Je résumerai ensuite I'histoire minière de cette région qui date d'un peu plus d'un siècle si l'on tient conpte de l'exploration. Dans une troisiène partie. je parlerai des inpacts que l'on trouve dans la région actuellement, ce qui constitue en un certain sens notre hérifage régional comme région-ressource. L'imaginaire et l'environnenent en seront longtemps narqués. Enfin, je présenterai trois sites touristiques originax et nouveaux qui sont de nature à démystifier quelque peu la géologie et l'exploitation des mines dans la région.

\section{BREF RAPPEL DE L'HISTOIRE- GÉOLOGIQUE ET DE LA VIE}

S'il y a une histoire d'exploitation minière en Abitibi-Témiscamingue au XXe siecle, c'est que des minéraux y ont été formés ou déposés il y a très longtemps, bien avant l'apparition de l'être humain sur la terre. Comme bien d'autres ressources naturelles, cet héritage a mis bien des millénaures alvan d'être découvert et utilisé.

L'écorce terrestre a commencé a se solidifier il y a quelque 4 milliards d'années, soit environ 600 millions d'années après la formation de la terre. Ces plaques solides qui formeront plus tard les continents, se déplaçaient sur le magma, se heurtaient, se soudaient ou changeaient de forme avant de se stabiliser quelque peu. Il y a quelque 2,7 milliards d'amées, des phẻnomenes de ce genre se sont produits en Abitibi'. Ces dérives de plaques tectoniques ont eu pour effet la formation des continents actuels, de montagnes et lat disparition de couches solides dans le magma interieur.

Parallèlement a la mise en place de cette structure physique, la vie prend sal place sur terre il y a quelque 3,5 milliards d'années sous forme d'algues unicellulaires sans noyau? Ici encore, la transformation se fait lentement et se continue de nos jours. Beaucoup plus près de nous, mais il y a quand même 600 millions d"années, la vie explose. Les poissons appanissent il y a trois cents millions d'années, les premiers êtres ầ se tenir debout il y a enwiron 5 millions d'années et beaucoup plus recenment, l'homme moderne, il y a moins de cent mille ans probablement.
Cet homme a su, peu à peu, apprivoiser et utiliser minéraux et autres especes vivantes pour survivre. L'histoire ancienne nous parle de mines exploitées pour le cuivre, l'or, l'argent. Cette exploitation est souvent illustrée par des récils d'exploitation humaine horribles.

Très récemment, il $\mathrm{y}$ a moins de cent ans, quelques-uns de ces êtres humains commencent à exploiter les minéraux de l'Abitibi-Témiscamingue. Il y a quelques années à peine, des gens ont voulu que cette exploitation soit mieux connue du public en général. Des moyens ont été pris pour que les touristes de la région et de l'extérieur puissent visualiser et vivre en quelque sorte, la vie des mineurs et mieux comprendre l'histoire géologique régionale. Voyons d'abord quelques faits de cette histoire minière.

\section{L'HISTOIRE MINIÈRE DE LA RÉGION}

Џ y a cent mille ans, à l'époque de l'apparition de l'homo sapiens sapiens, l'homme moderne, la région était de nouveau recouverte de glace et celle-ci n'a fondu qu'il $y$ a moins de dix mille ans. Les premiers habitants qui ont alors peuplé la région n'ont pas fouillé la terre en profondeur pour en tirer les métaux.

Ce n'est donc qu'avec l"arrivée récente des hommes blancs en Abitibi-Témiscamingue que les richesses minières ont été explorées à la fin du XIXe siècle et exploiteses sur une grande échelle à partir du début du XXe siecle, dans la 
foulée des découvertes minières faites auparavant dans le nord-est ontarien ${ }^{3}$.

Des intérêts financiers américains ont permis le developpement de ces mines ontariennes. Lorsqu' on s'est aperçu que la faille le long de laquelle se trouvait la majorité de ces mines se prolongeait au Québec, des intérêts financiers ontariens et autres se sont empressés de venir exploiter ces mines québécoises.

La première mine du Témiscamingue es! cependant connue depuis 1686 . Elle n'a été exploitée que plus tard, de 1890 à 1902 sous le nom de Wright. Les traces de cette exploitation ont longtemps été visibles, mais ont été effacées par la SOQUEM, il y a quelques années, apres avoir constaté la non-rentabilité des réserves de plomb.

Toutefois, l'exploration minière de la région a été entreprise activement à la fin du siecle dernier seulement. $\hat{A}$ cause de son isolement et du manque de moyens de transport facile, il faudra attendre les années 1920 pour les grandes découvertes et la mise en exploitation des gisements.

La mine Horne, exploitee par la Noranda Mines, fut la premiere et la plus importante mine de la région pour la production de cuivre, d'or et d'argent, avec ses 15 gisements. Elle entre en production en 1927. D'autres mines de la région RouynNoranda s'y ajoutent a partir de 1930. Des 1929, la mine Siscoe entre en production dans la région de Val d'Or. En 1950, 41 mines auront êt ́r mises en exploitation le long de la faille de Cadillac; 18 dans la région de RouynNoranda et 23 dans la région de Val d'Or. Une mine plus au nord s'est également ouverte à Normétal en 1937 et une plus au sud, a Belleterre, en 1935. De 1927 à 1950, l'Abitibi-Témiscamingue fournit la moitié de la valeur de la production minérale du Québec. En 1950, plus de 10000 travailleurs sont employés dans les mines de la région. Au cours de cetle epoque, les moyens de communication ferroviaires et routiers se multiplient. Des villes se construisent, la population augmente.

Par la suite, on assistera ane haisse des emplois miniers et $\mathrm{h}$ la fermeture de mines plus nombreuses qui sont épuisées ou devenues non rentables à cause des prix de l'or surtout. L'ouverture de mines dans la région de Chibouganáa, au nord, redonne l'espoir d'emplois plus réguliers et ouvre une nouvelle région d'exploitation de minéraux et de bois.

La mise sur pied de SOQUEM, la Société québécoise d'exploration minière en 1965, aura permis à des intérêts québécois de participer davantage à l'exploitation des richesses minières de la région. Au milieu des années 80, c'est la société privée CAMBIOR qui prendra la relève dans ce domaine. Depuis quelques années, la stabilité du prix de l'or et des autres minéraux permet au secteur minier une certaine stabilité.

\section{IMPACTS HUMAINS ET ENVIRONNEMENTAUX}

Historiquement, l'exploitation des mines à toujours cté associce h̀ l'exploitation humaine : travail forcé, danger constant, rếmunération faible. En Abitibi-Témiscamingue, la main d'oeuvre a toujours été abondante et volontaire, en grande partic parce que le chômage et la misère existaient ex existent encore largement dans la société québécoise et ailleurs dans le monde. Les accidents miniers ${ }^{4}$ et la faible rémunération des travailleurs ont toujours aussi été présents, laissant souvent des souvenirs amers, favorisunt la syndicalisition el des mouvements de contestation.

L'exploration, la découverte el la mise en valeur demandent beaucoup d'argent. Whe partie de cet argent a ćté amasséc par la vente de aparts de minesw. Plusieurs individus ou fanilles ont ainsi êté ruinés par des vendeurs habiles de aparts de mines qui n'ont jamais existé ou qui ne sont jamais parvenues à l'élape d'exploitation remable.

Ce n'est qu"assez réccemment que la population a pu influencer les gouvernements et les industries minieres alin que les méthodes de transformation des mélaux soient moins dommageables pour l'environnement. Des pertes énormes pour des individus et la collectivité ont été ainsi occasionnês et ont réduit considérablement la qualité de vie. L'air, l'eau et la terre pollués ne sont plus aussi accessibles, utiles el agréables que par le passe er parfois carrément inutilisables et dangereux. Des efforts sont fiuts pour remédier a la situation, mais le plus gros reste à faire et l'exploitation sans pollution est encore loin d'être chose du passé. L'idée que les ressources naturelles sont des richesses collectives et doivent servir la population et pas seulement les exploitants et les gouvemements est une idée relativement récente, ainsi que l'idée de responsabilité des exploitants face aux dégâts environnementaux. Mais peu à peu, ces idées font leur chemin.

Les fermetures de mines, pour des causes naturelles comme l"épuisement des gisements ou plus contextuelles comme la baisse de la demande ou des prix, ou encore à cause des conflits syndicaux, ont souvent laissé des travailleurs et leurs familles dans des conditions de misère. Les villages fantômes se sont multipliés. Des iravailleurs qui avaient investi leur revenus dans l'achat d'une maison se retrouvent, à un âge parfois avancé, avec une propriété qui n'a presque plus de valeur. Il est parfois difficile, sinon impossible de se relocaliser. Chômage, bien-être social et misere sont trop souvent au rendez-vous.

Les profits souvent énormes réalisés par les propriétaires sont la plupart du temps investis hors de la région. Cela est vai aussi pour d'autres ressources naturelles d'ici. Vivre dans une région-ressource laisse le sentiment de travailler pour les autres et d'avoir peu de possibilités de préparer un avenir confortable. La perte de revenu peut survenir de façon imprévisible et personne ne semble se préoccuper de trouver un nouvel emploi a la main d'oeuvre mise à pied. La population accepte mal qu'une richesse collective n'enrichisse, en bout de ligne, que les propriétaires de quelques grandes compagnies.

Comme on peut le voir, si l'exploitation et la mise en valeur des métaux sont nécessaires au progrès de l'industrie, les travailleurs miniers sont susceptibles de rencontrer bien des difficultes. Les régions minières deviennent rapidement des lieux pollues, les profits sont souvent réinvestis ailleurs. La population qui reste sur le territoire, souvent dévasté et inutilisable, ne peut bénéficier que rarement des retombées positives à long terme et se retrouve souvent au point de départ et avec peu de possibilités de recommencer sur place ou ailleurs.

Comme dans toute région-ressource, l'é- 
conomie de la région est dépendante pour une large part de décisions et de financements venant d'ailleurs. L'industrie secondaire, encore embryonnaire, est également souvent contrôlée par des intếrêts et un marché extérieurs qui rend ainsi la population très dépendante et insécure.

Céla est vrai, non seulement dans le domaine minier, mais également dans le domaine forestier, agricole et hydroélectrique. Dans tous les domaines, les travailleurs, un jour ou $\mathrm{l}^{*}$ autre, ont le sentiment de «s'être fait avoir», d'avoir peiné pour les autres pendant de nombreuses années et d'en avoir peu retiré. Ce qui malheureusement est souvent le cas.

Malgré tous ces aspects négatifs de l'hếritage régional actuel, une bonne partie de la population a décidé de vivre dans cette région et s'y est attachée. Depuis vingt-cing ans surtout, des efforts énormes sont consacrés a tenter de stabiliser les emplois, de développer des services, d'agir en vue de diminuer la pollution et de réparer les dommages faits a $l^{\prime}$ environnement; en un mot, on essaie de faire de l'Abitibi-Témiscamingue, une région où il fait bon vivre. Les mouvements de contestations, de demandes pour plus de services, de prise en charge et d'organisation se sont multipliés dans tous les domaines. Le bénévolat fleurit.

Le développement d"attraits touristiques. fait partie de cet effort de prise en main de développement. Plusieurs musées et lieux intếressants sont maintenant à la portêe du touriste un peu partout en région, grâce à des efforts soutenus de personnes et de groupes dynamiques. Au point de vue geologique et minier, qui nous intéresse ici en particulier, on peut mentionner trois endroits qui altirent et intéressent de plus en plus le voyageur provenant de l'intérieur ou de l'extérieur de la région. Il s"agit de la Cité de l"Or à Val d'Or, du musée régional des mines à Malartic, et du musée des fossiles at Notre-Dame-du-Nord.

\section{QUELQUES ATTRAITS TOURISTIQUES ISSUS DE LA GÉOLOGIE ET DES MINES}

En Abitibi-Témiscamingue, en plus de la grande nature qui altire bien des amateur's de plein air, des sites historiques nom-

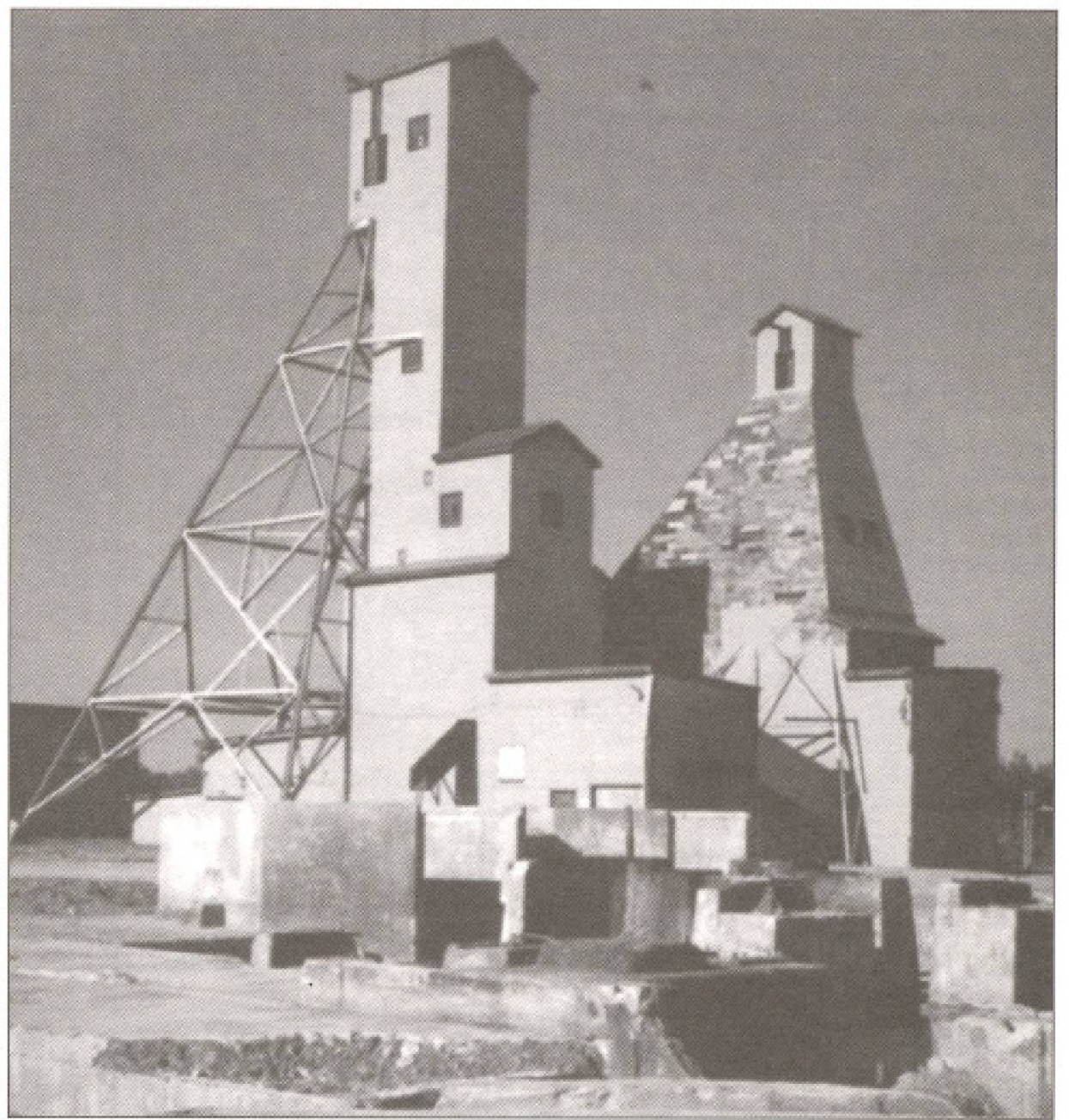

Chevalenents de la wine Lanaque (carte postale distribuée par la Cité de l'Or et la Corporution du Village minier de Bourlaniaque).

breux son! offerts áux visiteurs intéressés at l'histoire agricole, commerciale, forestière, miniere ou culturelle de la région. Trois de ces siles proposent aux visiteurs de mieux comaître la géologie et l'exploitation minière. La Cité de l'Or; at Val-d'Or, propose atux visileurs de vivre lat vie d'un mineur pour quelques heures. A Matartic, le touriste péul se renseigner sur les domaines liscinaunds de lá géologie el de la mincralogie et â Notre-Dane-duNord, de nombreux exibits et tablenux expliquent en partie lá géologie de la région du Témiscanningue en présentant une exposition sur des fossiles. Trois endroils où les surprises, les apprentissages et l'intérêt ne manquent pass. Le monde mystérieux de la croûte terrestre et de l'exploilation des richesses qu'elle contient deviennent de plus en plus accessible at toutes les personnes curieuses qui s'intéressent ho cos sujets.

\section{LA CITE DE L'OR}

Un touriste peut facilement visiter la région de l'Abitibi-Témiscamingue sans voir beaucoup de choses au sujet des mines. Il wroule sur l'ors, mais il n"en a guère conscience. De plus, le travail sous terre, ce lieu mystérieux et sombre, est très mal connu et la demande des visiteurs pour descendre dans une mine est tres forte. Peu de visiteurs, jusqu"à récemment, pouvaient satisfaire facilement cette curiosité légitime.

\section{LA CITÉ DE L'OR VIENT COMBLER CE BESOIN DE CONNAITTRE DEPUIS 1995.}

De nombreuses personnes et organismes se sont joints à la "Corporation du Villaye minier de Bourlamaque", dont I'Ollice du Tourisme de Val-d'Or depuis 
L'ABITIBI-TÉMISCAMINGUE

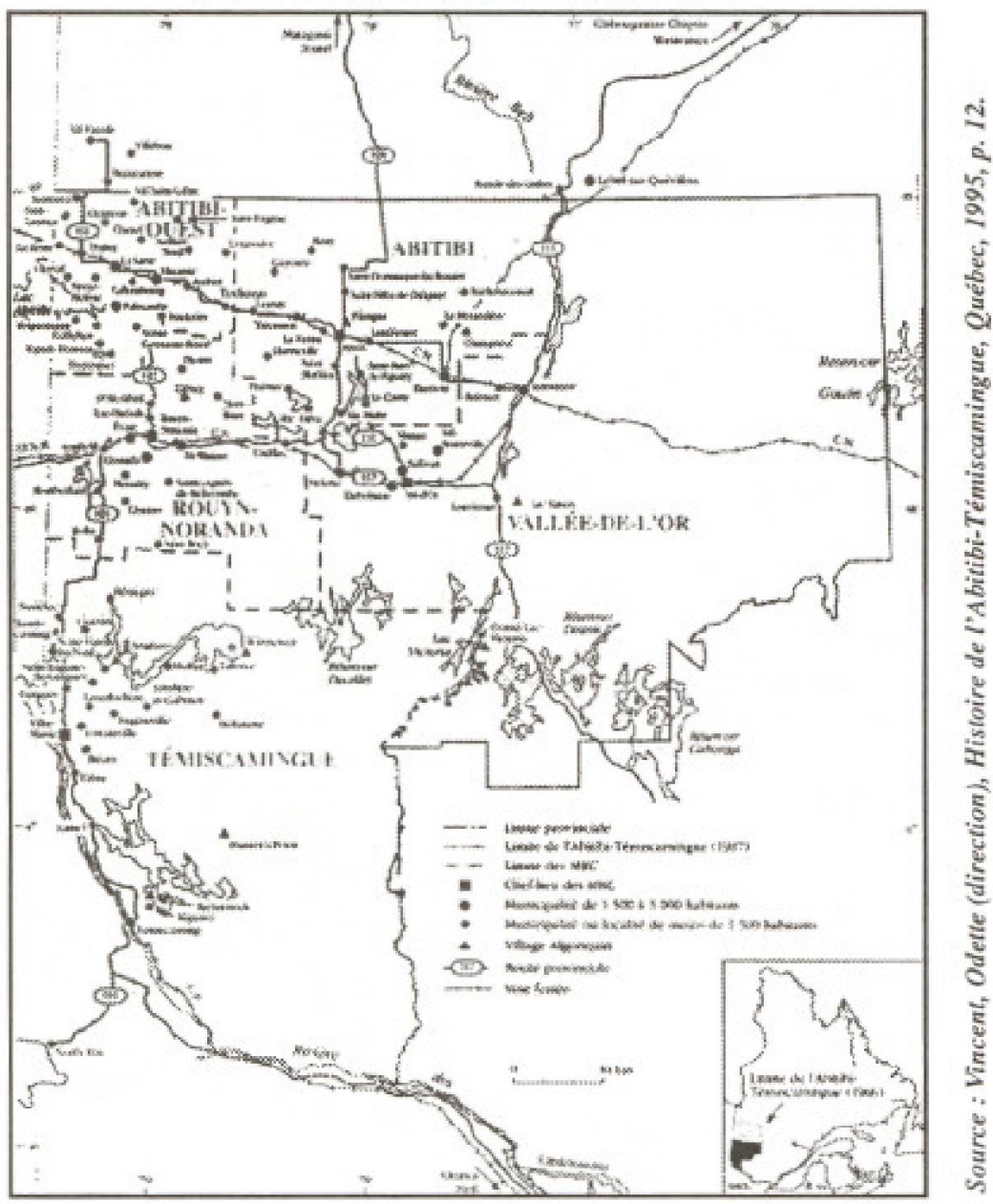

plus de 10 ans, pour enfin en arriver à un concept des plus intéressants.

Dess cette première année d'ouverture au grand public, plus de 10000 visiteurs sont venus profiter des trois sites offerts: d'abord, la visite de la galerie du troisième niveau a 80 mètres (264 pieds) sous terre où on accède par une rampe à 15 degrés d'inclinaison. Le visiteur aura préalablement revêtu de vrais habits de mineur et c'est ainsi accoutré qu'il pourra, avec un guide, visiter les 26 exibits fournis par differentes entreprises minières qui sont étalés sur 1,5 kilomètre. Tout cela à la lumiere artificielle et a une température constante d'environ 11 degrés C.

Les bâtiments de surface sont aussi visibles et on peut en visiter quelques-uns. La mine Lamaque a la particularité unique d'avoir deux chevalements. L'un d'eux a brûlé lors de la réfection du toit. La structure est cependant encore en place et sera repeinte. Une attraction supplémentaire! Enfin, troisième activité, la visile d'une des 80 maisons du village minier de Bourlamaque construites en 1934 dont 79 sont encore louées et habitées.

La Cité de l'Or est un centre d'interprétation unique en Amérique du Nord. Les visiteurs peuvent y apprendre une foule de choses intéressantes devenues parties du patrimoine minier, comme l'histoire de la mine Lamaque, dont les actionnaires ont investi des millions de dollars pour la construction du village et le chevalement. La mine, la plus riche de la région à l'époque, a récupéré ses investissements en trois ans. De 1935 à 1985 , plus de 26 millions de tonnes de minerai sont extraites et donneront 4,5 millions d'onces d'or ou environ 4500 lingors.

Un des nombreux panneaux de renseignement vous apprendra que la faille de Cadillac est une bande de roches tendres et fractureses, vicilles de 2,5 milliards d'années dans laquelle se sont infiltrées des roches dures, porteuses d'or. Vous saurez aussi que la veine aurifere, cause de cette activité minière, a été trouvée par Robert Clark en 1923 et qu'il était accompagné par l'Amérindien Gabriel Commanda. Cette mine est situce dans le canton Bourlamaque, ainsi nommé comme bien d'autres lieux abitibiens, a partir des noms de soldats français venus au Canada sous les ordres de Montcalm et Lévis au XVIIIe siècle.

Ce vếcu d'une journée dans la vie d'un mineur laisse des souvenirs irremplaçables et uniques. Je m'arrête ici, faute d'espace et pour laisser aux visiteurs le plaisir de nombreuses autres découvertes.

\section{LE MUSÉE RÉGIONAL DES MINES DE MALARTIC ${ }^{\circ}$}

Situé à quelques kilometres de Val-d'Or, ce musée a été le premier à offrir au public un centre d'interprétation d'activités minières comprenant l'exploitation et la transformation et ce, depuis 1982 . Une simulation de descente dans une mine conduisait à differents sites où le visiteur pouvait se faire une idee du travail du mineur. Avec l'ouverture de la Cité de l'Or qui offre une amélioration de cette activité, le musée de Malartic change de vocation et devient un musée de minéralogie et de géologie. L'accent sera mis sur la région, mais des exibits nombreux venant d'autres régions du monde seront visibles egalement.

La présentation se fera sous forme de théâtre de contextualisation dans lequel le visiteur sera amené (sans guide humain) a se familiariser avec les differentes utilisations que les hommes font des minéraux, de la pierre utilisée pour sa beauté ou son utilité par différentes catégories de collectionneurs ou d'utilisateurs. Plusieurs média seront utilisés. Le visiteur deviendra un acteur dans différents décors. Il pourra manipuler certains objets. On y trouvera même la diseuse de bonne aventure.

Le musée sera ouvert toute l'année. Sa vocation éducative se concrétisera en particulier par un parcours pédagogique, "Aventuriers de la roche perdue", s'adressant particulièrement aux enfants du primaire. 
Un tout nouveau concept pour la région, qui ne manquera pas d'attirer de nombreux visiteurs intéressés à la géologie et aux roches de la région et d'ailleurs. On prévoit également changer la présentation et une partie des nombreux exibits d'une année à l'autre.

\section{L'EXPOSITION DU}

\section{REGROUPEMENT DES LOISIRS CULTURELS ET SCIENTIFIQUES DU TÉMISCAMINGUE À NOTRE-DAME- DU.NORD.}

Plus au sud de la région, le touriste peut découvrir d'autres activités géologiques et minières. Entre Malartic et NotreDame-du-Nord, à Rouyn-Noranda, Minéraux Noranda offre une visite qui renseigne sur la transformation du minerai et la récupération.

A Notre-Dame-du-Nord, plus au sud, le Témiscamingue présente une exposition connue depuis 1987 sous le nom de "Nos ancêtres de 400 millions d'années". Cette activité tire son origine de l'intérêt montré par Andrée Nault lors de ses études de géologie à l'UQAM pour la paléontologie qui l'a amenée à étudier et à collectionner de nombreux fossiles témiscamiens?. Avec l'aide de la Société d'Histoire du Témiscamingue, une exposition de ces fossiles fut montée en 1987 , attirant près de 4000 visiteurs. Pour expliquer la présence de ces fossikes de région tropicale au Témiscamingue, une attention spéciale est portée a $1^{\top}$ histoire géologique de la région. Une brochure sur le sujet fut éditée en 1988. Chaque année, depuis ce temps, une exposition dans des locaux temporaires à NotreDame-du-Nord a un grand succès el la collection s'enrichit continuellement de fossiles et de roches expliquant l'histoire géologique du Témiscamingue.

Devenue propriété de la Sociéıé d'Histoire en 1987, le Comité touristique de la Chambre de Commerce de NotreDame-du-Nord en assume la mise en valeur depuis 1990 grâce au dévouement des membres d'un sous-comité (Comité des loisirs culturels et scientifiques du Tếmiscamingue) créé en 1991. (irîce à de nombreuses subventions de provenances locales et gouvernementales, un centre d'exposition permanent devrait voir le jour en 1998 . Le comité responsable organise également de nombreuses sorties au cours desquelles des notions de géologie et de paléontologie sont acquises par les participants. De nouveaux échantillons s'ajoutent à la collection par la même occasion. $\grave{A}$ visiter en passant à Notre-Dame-du-Nord.

\section{CONCLUSION}

L'Abitibi-Témiscamingue demeure pour beaucoup de personnes de l'extérieur un lieu éloigné et étrange. L'exploitation des ressources naturelles s'y est faite sur une grande échelle (fourrures, forêts et mines surtout) avec une population dispersée sur un grand territoire.

Les résidents permanents de la région tra= vaillent depuis longtemps à diversifier les activités et à offrir plus de services. Le tourisme est un secteur actuellement en plein développement. La géologie et les mines de la région offrent ainsi des thèmes qui sont de plus en plus mis en valeur et s'ajoutent aux nombreux autres attraits de la région pour le plus grand intêrêt et le plus grand plaisir du touriste.

\section{NOTES BIBLIOGRAPHIQUES}

1 Dans un dossier d'Isabelle Montpetit "Les dix découvertes de l'année" (Québec Sicience, wol 34, no 5, fovnic 1996), aux pages 26-27, on peut apprendre comment Andrew Calvet et son equipe ont pu mieux comprendre la géologie d'un coin de la région abitibienne, au sud de Malagami, en prouvant que la dérive des continents existait à cetle êpoque.

2 Une bonne partie des énoncés de celle seetion du texte s"inspire de l'article "When life exploded" de Madeleine Nash paru dans le Time du 4 decembre 1995, p. 36-46.

3 Differentes sections du volume Histoire de l'Abiribi-Témiscamingue, collectif ecrit sous la direction d'Odente Vincent (Quihes, IQRC, 1995) ont th consultes pour et aticle.

4 Dans un article de La Froniëre du 3 juin 1992, Camille Beaulieu mentionne qu'il y avait eu 612 accidents ninicrs au Québec depuis 1936, selon L'Association minitre du Quéthes. Cette derniêre "n"a créć qu'en 1948, son Comité de prévention des accidents".

5 Les renseigneuents sur la Cité de l'Or viennent d'une visite que j"y ai faite le 23 fevrier 1996. Suzanne Pepin $\mathrm{m}$ 'a fait visiter l'ćlifice ouvent loul I'hiver at in' is fourni une bonne documentation : articles divers, la brochure "Dans c'temps-lä, Bourlamaque"... et a aimablement répondu à mes nombreuses questions. J'ai également consulté le volume de la Société d'Histoire de Val-d'Or intitulé "Histoire de Val-d'Or des origines à 1995", (Val-d'Or, 1995).

6 Mes informations viennent principalement de Pierre Routhier que j'ai rencontré au Muscé alors en phase de transformation, le 15 mars 1996. II a aimablement répondu à toutes mes questions, mais ne peut être tenu responsable des interprétations que je peux en faire.

7 Les renseignements sur cette partie du texte viennent de diverses sources : conversations, documents divers et particulièrement : Nault, Andrée et Michel Di Vergilio, Chez nos ancêtres de 400 millions d'années, (Ville-Marie, Société d'Histoire du Térniscamingue, 1988); l'article sur le "Regroupement des Loisirs culturels et scientifiques du Témiscamingue" dans NotreDame-du-Nord 1896-1996 (Mare Riopel et le Connité du Livre, 1995, p. 217)

8 Annonce faite dans Le mot qui court du 21 mars 1996 sous la signature de Thérèse Aylwin et de Jocelyne Pillon, présidente et publiciste du Regroupement des Loisirs culturels et scientifiques du Témiscamingue, wLes visiteurs pourront marcher dans le décor recrés de cette ancienne mer (tropicale), voir ces animaux (aujourd'hui fossillisés) vivie leur vie, se nourrir et mourirm. peut-on lire dans l'annonce de ce projet. 